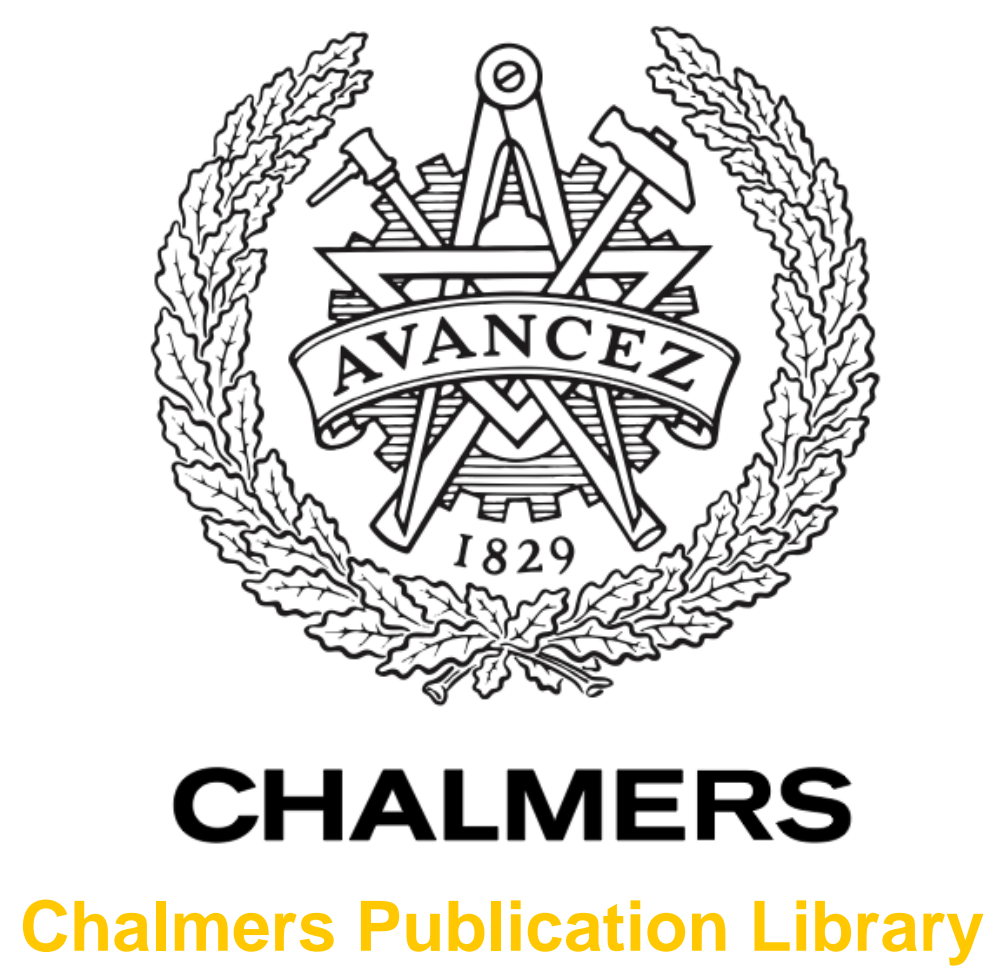

\title{
Concentrator of magnetic field of light
}

This document has been downloaded from Chalmers Publication Library (CPL). It is the author's version of a work that was accepted for publication in:

Proceedings of SPIE - Conference on Metamaterials VII, Brussels, 16-19 April 2012 (ISSN: 0277-786X)

Citation for the published paper:

Wróbel, P. ; Stefaniuk, T. ; Antosiewicz, T. (2012) "Concentrator of magnetic field of light". Proceedings of SPIE - Conference on Metamaterials VII, Brussels, 16-19 April 2012, vol. 8423

http://dx.doi.org/10.1117/12.922912

Downloaded from: http://publications.lib.chalmers.se/publication/163049

Notice: Changes introduced as a result of publishing processes such as copy-editing and formatting may not be reflected in this document. For a definitive version of this work, please refer to the published source. Please note that access to the published version might require a subscription.

Chalmers Publication Library (CPL) offers the possibility of retrieving research publications produced at Chalmers University of Technology. It covers all types of publications: articles, dissertations, licentiate theses, masters theses, conference papers, reports etc. Since 2006 it is the official tool for Chalmers official publication statistics. To ensure that Chalmers research results are disseminated as widely as possible, an Open Access Policy has been adopted.

The CPL service is administrated and maintained by Chalmers Library. 


\title{
Concentrator of magnetic field of light
}

\author{
Piotr Wróbel*a ${ }^{\mathrm{a}}$, Tomasz Stefaniuk ${ }^{\mathrm{a}}$, Tomasz J. Antosiewicz ${ }^{\mathrm{b}}$, Tomasz Szoplik ${ }^{\mathrm{a}}$ \\ ${ }^{a}$ University of Warsaw, Faculty of Physics, Pasteura 7 Str., 02-093 Warsaw, Poland \\ ${ }^{\mathrm{b}}$ Chalmers University of Technology, Department of Applied Physics, SE-412 96 Goteborg, \\ Sweden.
}

\begin{abstract}
In the recent decade metamaterials with magnetic permeability different than unity and unusual response to the magnetic field of incident light have been intensively explored. Existence of magnetic artificial materials created an interest in a scanning near-field magnetic microscope for studies of magnetic responses of subwavelength elementary cells of those metamaterials. We present a method of measuring magnetic responses of such elementary cells within a wide range of optical frequencies with single probes of two types. The first type probe is made of a tapered silica fiber with radial metal stripes separated by equidistant slits of constant angular width. The second type probe is similar to metal coated, corrugated, tapered fiber apertured SNOM probe, but in this case corrugations are radially oriented. Both types of probes have internal illumination with azimuthally polarized light. In the near-field they concentrate into a subwavelength spot the longitudinal magnetic field component which is much stronger than the perpendicular electric one.
\end{abstract}

Keywords: optical magnetism, plasmonics, magnetic metamaterials, scanning near-field magnetic microscopy, corrugated SNOM probes.

\section{INTRODUCTION}

Pendry et al. [1] have shown that artificial materials composed of elementary cells fabricated from nonmagnetic conducting elements have effective magnetic permeability different from unity. Urzhumov and Shvets [2] presented theoretical formulas for the frequencies and strengths of electric and magnetic resonances valid for any periodic metallodielectric nanostructures operating in the plasmonic regime. More recently, Merlin [3] considered a metamaterial composed of split rings and spherical inclusions. He showed that if a metamaterial is made of substances with the $\operatorname{Im}\left|\sqrt{ } \varepsilon_{\mathrm{m}}\right|>>\lambda / \mathrm{d}$, where $\varepsilon_{\mathrm{m}}$ is the complex permittivity of the metamaterial elementary cell and $\lambda>>d$ is the wavelength in vacuum and $d$ is the characteristic length of the cells, then strong diamagnetic or paramagnetic behaviour characterized by susceptibilities whose magnitude is significantly larger than that of natural substances is possible. According to Merlin, the strength of magnetic effects diminishes with decreasing wavelength. Existence of metamaterials composed of subwavelength elementary cells made of materials with large values of the permittivity creates a need for an analogue of a scanning near-field optical microscope (SNOM) where in search of a magnetic response the cell is illuminated with a concentrated longitudinal component of magnetic field of electromagnetic wave.

The idea of scanning near-field magnetic microscope (SNMM) can be realized in different ways. In the inverse transmission mode of work of SNOM the probe does not serve as a source of evanescent optical field but as a local detector of light. Using this mode of work Devaux et al. [4] observed that the fully dielectric tapered probe collects a signal proportional to the square modulus of the electric near field, while the tapered gold-coated 'display the same patterns as the theoretical maps of the square modulus of the magnetic field associated with the optical near field.' Burresi et al. [5] have reported on SNOM detection of the near-field magnetic component of light in phase-sensitive heterodyne scheme, where signal from an aperture regular probe is combined with that from a probe with split ring resonator (SRR) in the aperture plane. Aperture probes without an SRR at the tip end were used for passive measurements of the magnetic component of modes excited in photonic crystal cavities coupled to waveguides [6-7]. Banzer et al. [8] proposed an optical set-up for testing electric and magnetic properties of single sub-wavelength nanostructures. As an example, the electric and magnetic resonances of a properly oriented SRR were independently measured by means of a y-polarized $\mathrm{TEM}_{10}$ mode (y-polarized $\mathrm{HG}_{01}$ mode) with a strongly focused on-axis longitudinal magnetic field $\mathrm{H}_{\mathrm{z}}$ and off-axis $\mathrm{E}_{\mathrm{y}}$ components.

* e-mail: piotr.wrobel@igf.fuw.edu.pl; Tel: +48 225546896.

Metamaterials VII, edited by Allan D. Boardman, Nigel P. Johnson, Richard W. Ziolkowski, Proc. of SPIE Vol. 8423, 84231T · @ 2012 SPIE · CCC code: 0277-786X/12/\$18 · doi: 10.1117/12.922912 
Recently, Kihm et al. [9] attracted attention to the fact that when a single subwavelength aperture in a metal plane is illuminated with linearly polarized light at an oblique incidence then the surface electric field and surface current are mostly induced by the incident magnetic component of electromagnetic field. Thus, normal-angle scattering from a subwavelength aperture is governed by the magnetic field component of light and thus the aperture acts as a polarization analyser for magnetic vector field.

In this technical note we present two types of tapered along the $\mathrm{z}$ direction dielectric probes with discrete $[10,11]$ or continuous metal coating which concentrate longitudinal magnetic field of light in the near field of their apexes. Cross sections of the probes are shown in Figure 1(left) and (right) - the larger pictures show the xy plane cross sections far away from the apex, while the smaller ones at the apex. The probes have internal azimuthally polarized illumination coupled to plasmons due to momentum matching on radial gratings. The gratings have continuously changing period what allows for efficient coupling within a wide spectral range. Plasmons with a strong azimuthal component propagate towards the apex where they form an azimuthal current in the metal which results in generation of a strong longitudinal magnetic field $\mathrm{Hz}$.

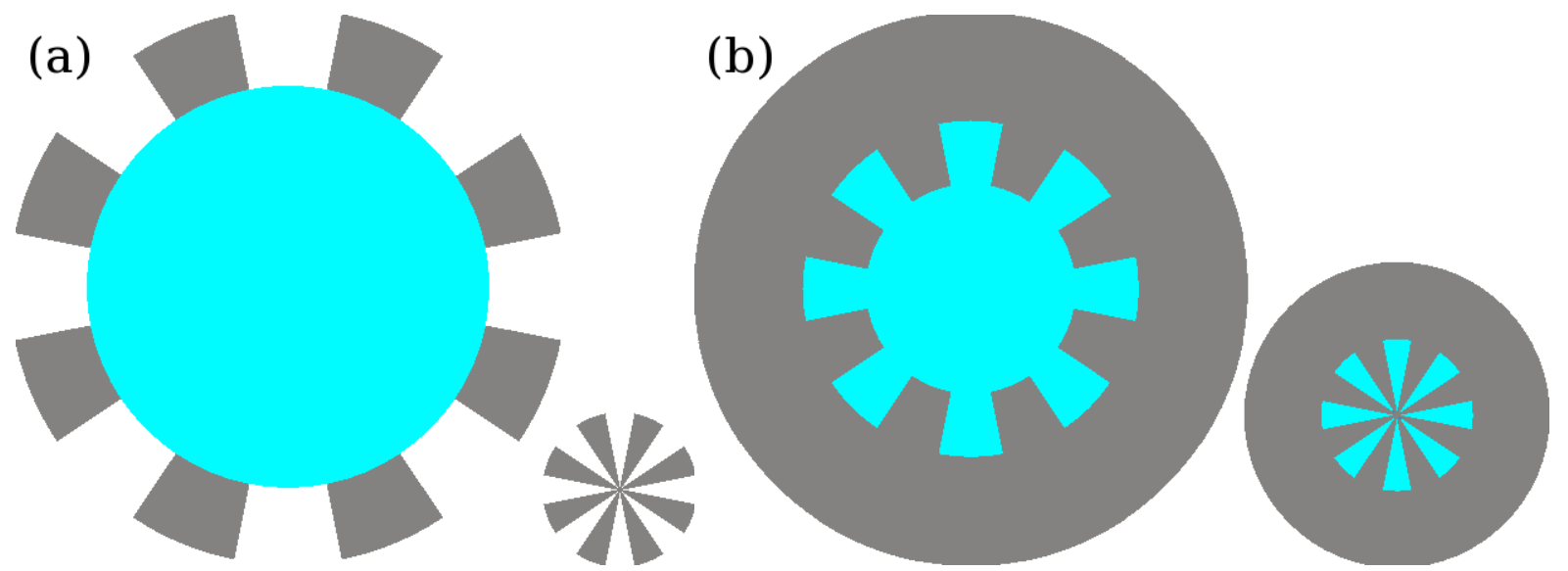

Figure 1. (left) Cross sections of dielectric probe with metal stripes: large graphic shows a cross section far from the apex, small: at apex. The metal thickness of the metal stripes is constant. (right) Cross sections of metal-coated dielectric probe with radial groves: large graphic shows a xross section far from the apex; small at apex. The metal coating thickness as well as groove depth are constant.

Recently, in an effort to find an easy method to fabricate magnetic field concentrators other geometries were proposed [12]. Lee et al. [12] use external illumination and grating-coupling to surface plasmons onto metallic tips. Concentration of $\mathrm{H}_{\text {apex }}$-field and at the same time strong suppression of $\mathrm{E}_{\mathrm{z}}$-field is achieved for double-sided illumination in the the $\mathrm{H}$ symmetric (E out-of-phase, $\mathrm{H}$ in-phase) or E-antisymmetric (E field out-of phase, $\mathrm{H}$ field in-phase) surface plasmon excitation schemes.

\section{TAPERED DIELECTRIC PROBES WITH RADIAL STRIPES}

Magnetic concentrators of the first type are made of dielectric nondispersive fibers with core diameter equal $3.2 \mu \mathrm{m}$ which taper smoothly from their regular diameter to the apex. In the simulations we accept a taper half-angle equal to $40^{\circ}$. The metal lands and the slits have constant angular width equal $\pi / 8$ (eight periods total). The metal lands are made of $\mathrm{Ag}$ or $\mathrm{Al}$ with thicknesses $\mathrm{h}$ varied from 0 to $100 \mathrm{~nm}$. They are modeled using Drude dispersion $\varepsilon(\omega)=\varepsilon_{\infty}-\omega_{\mathrm{p}}^{2} /[\omega(\omega$ $+\mathrm{i} \Gamma)$ ] fitted to experiental data obtained by Johnson and Christy [13] for Ag with parameters equal $\varepsilon_{\infty}=3.70, \omega_{\mathrm{p}}=13673$ $\mathrm{THz}$, and $\Gamma=27.35 \mathrm{THz}$. For Al we use data from Ordal et al. [14] with fitting parameters $\varepsilon_{\infty}=4.39, \omega_{\mathrm{p}}=12062 \mathrm{THz}$, and $\Gamma=1009 \mathrm{THz}$.

Figures 2 and 3 show full-width at half-maximum (FWHM) of the longitudinal magnetic field component $\mathrm{Hz}$ calculated $10 \mathrm{~nm}$ from the apexes of probes with $\mathrm{Ag}$ and $\mathrm{Al}$ stripes, respectively. In both cases the stripe thickness changes from 0 to $100 \mathrm{~nm}$. For the largest thickness considered FWHM values are better than half a wavelength. In the case of thin metal stripes plasmons radiate into an azimuthally polarized beam before reaching the apex and diffraction enlarges the spot size considerably. In the whole range of wavelengths FWHM values available with Al stripes monotonically grow with increasing wavelength. This regularity suggest that aluminum is a better choice for stripe metal than silver. 


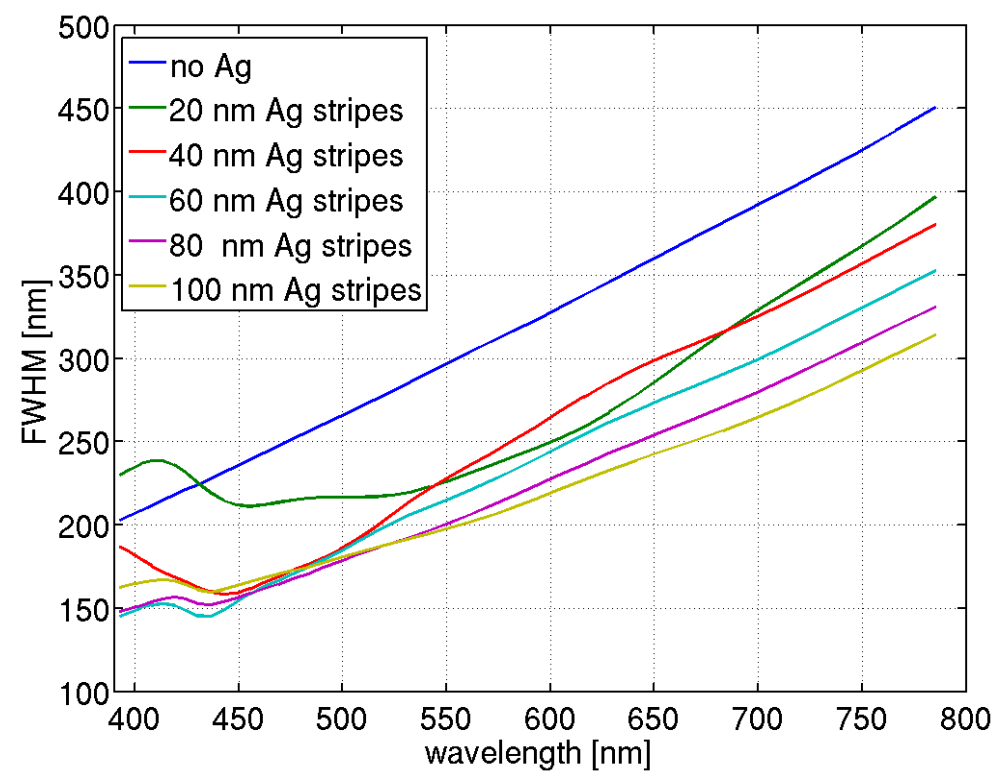

Figure 2. Full-width at half-maximum of the longitudinal field component $\mathrm{Hz}$ calculated $10 \mathrm{~nm}$ from the apex of a dielectric probe with silver stripes.

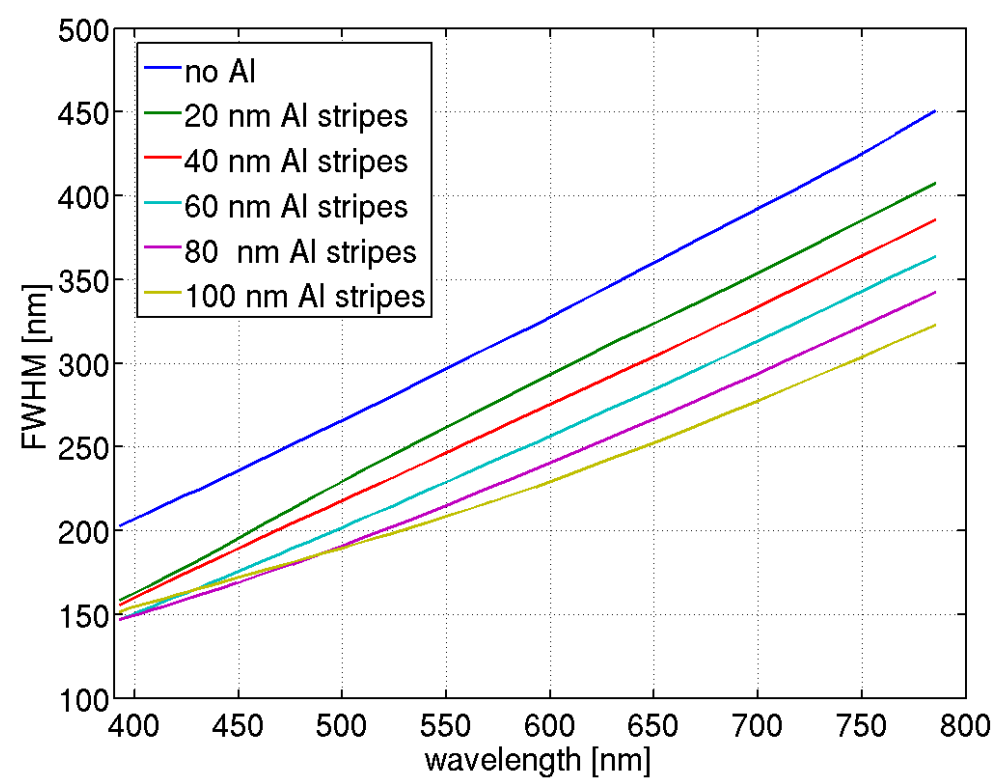

Figure 3. Full-width at half-maximum of the longitudinal field component $\mathrm{Hz}$ calculated $10 \mathrm{~nm}$ from the apex of a dielectric probe with aluminum stripes.

In near-field of probe apexes not only the longitudinal, accumulated at the z-axis, component of magnetic field is present but also an azimuthal component of the electric field with its energy density increasing away from the z-axis. Figures 4 and 5 present a ratio of magnetic energy density of $\mathrm{Hz}$ and electric energy density of $\mathrm{E} \varphi$ integrated over an area of diameter equal to the FWHM of $\mathrm{Hz}$ for $\mathrm{Ag}$ and $\mathrm{Al}$ probes, respectively. In probes with silver stripes this magnetic-toelectric energy density ratio is higher than in those with $\mathrm{Al}$ stripes. It is because a narrow $\mathrm{Hz}$ needle is generated by azimuthal currents $\mathrm{J} \varphi$ flowing through the set of stripes and virtual displacement currents in grooves. Those currents are larger in silver which is less lossy than aluminum. For both metals the optimum stripe thickness is within the range from 40 to $100 \mathrm{~nm}$. 


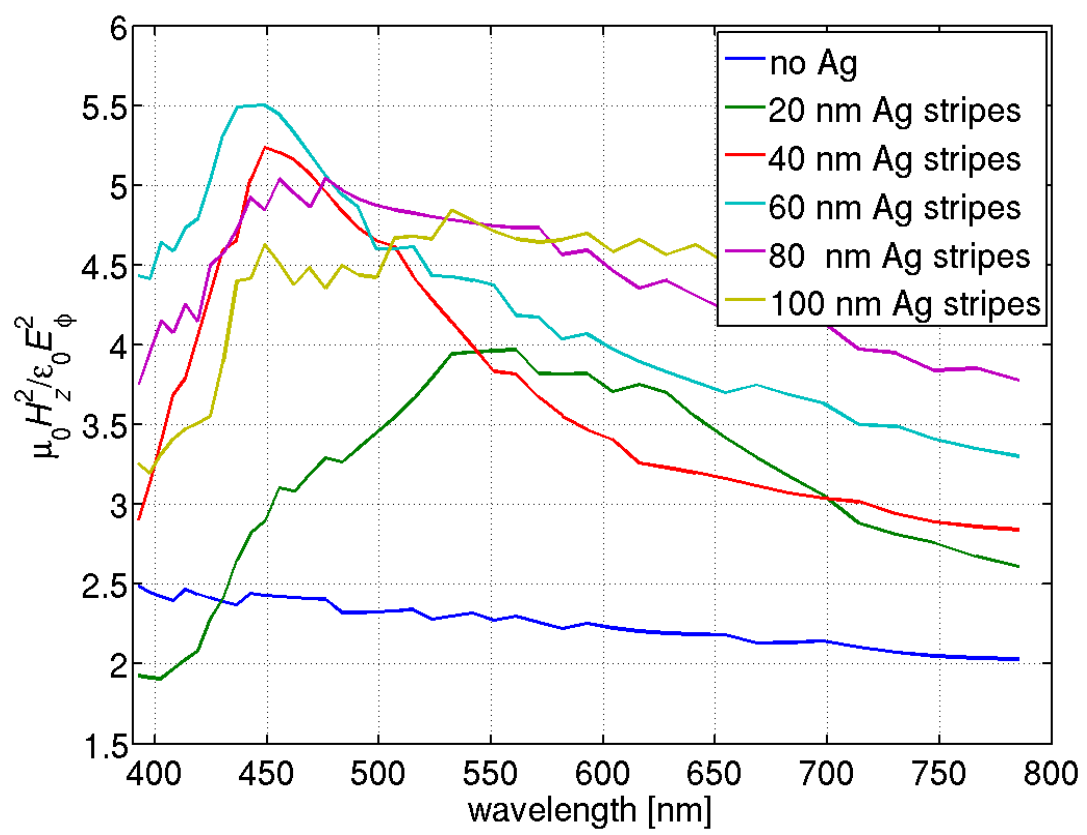

Figure 4. Ratio of magnetic energy density of $\mathrm{Hz}$ to electric energy density of $\mathrm{E} \varphi$ integrated over an area of diameter equal to the FWHM of $\mathrm{Hz}$ for dielectric probes with silver stripes.

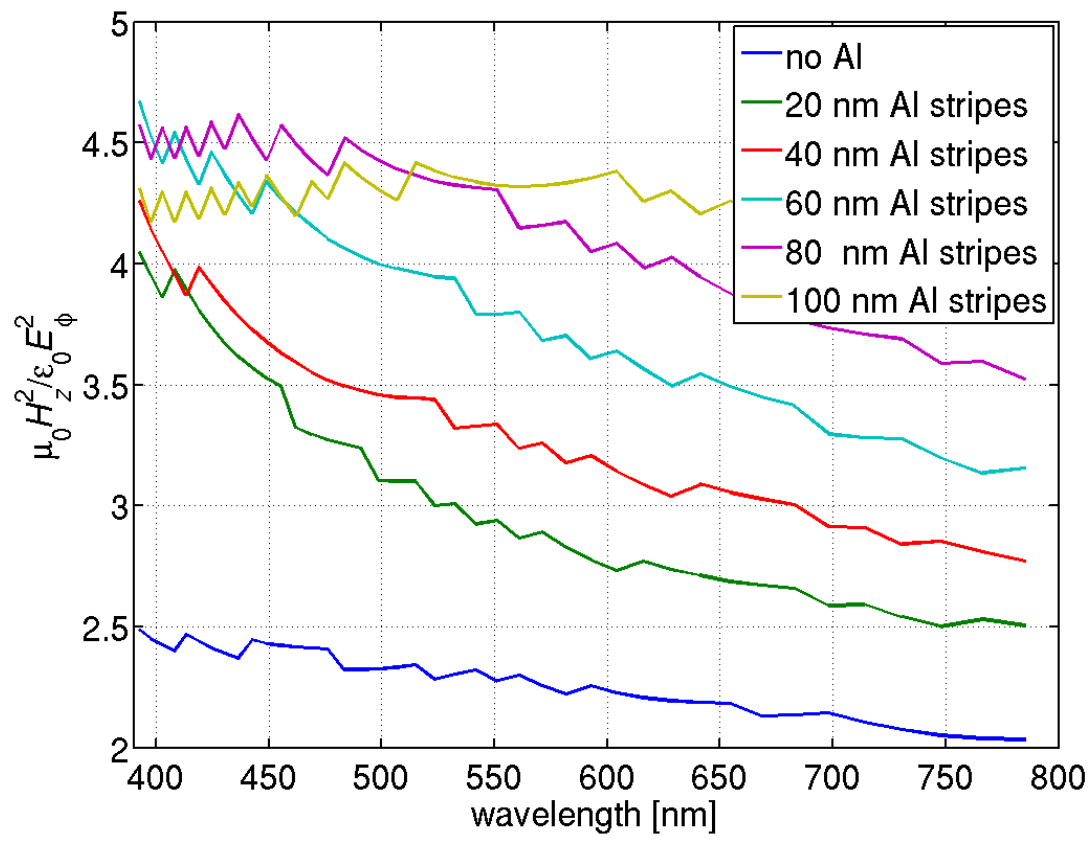

Figure 5. Ratio of magnetic energy density of $\mathrm{Hz}$ to electric energy density of $\mathrm{E} \varphi$ integrated over an area of diameter equal to the FWHM of $\mathrm{Hz}$ for dielectric probes with silver stripes.

Fabrication of probes with radial metal stripes completely separated by narrowing along the probes grooves is not an easy task and makes use of focused ion beam etching. Below we propose another geometry of a concentrator of longitudinal magnetic field component of light. 


\section{TAPERED DIELECTRIC METAL COATED RADIALLY CORRUGATED PROBE}

Currently we fabricate SNOM probes in the form of apertured metal-coated tapered fibers where light propagating in the fiber core is coupled to surface plasmons on corrugated core-metal coating interface [15]. When corrugations are made on the tapered fibre surface along the $\mathrm{z}$ axis, in simulations we use internal linearly polarized illumination what reduces the problem to two dimensions [16-18]. Real probes have internal illumination with radial polarisation.

The longitudinal magnetic field component $\mathrm{Hz}$ can be generated with azimuthal current $\mathrm{J} \varphi$ on the edge of continuous metal coating at the apex of aperture probe. This is possible when internal azimuthally polarized illumination is coupled to plasmons on core-metal coating interface with corrugations along the angular coordinate (Figure 1b). Simulated performance of such a probe with $\mathrm{Al}$ coating in presented in Figures 6 and 7. FWHM of the longitudinal magnetic field component $\mathrm{Hz}$ calculated $10 \mathrm{~nm}$ from the apex becomes narrower than that of uncorrugated probes for grooves $40 \mathrm{~nm}$ deep and more. In comparison with results shown in Figures 2 and 3 the radially corrugated probes gives better FWHM values especially for short wavelengths. Figure 7 shows that ratio of magnetic energy density of $\mathrm{Hz}$ to electric energy density of $\mathrm{E} \varphi$ integrated over an area of diameter equal to the FWHM of $\mathrm{Hz}$ is is virtually independent on depth of grooves. The ratio reaches values one order of magnitude bigger than those possible with probes with radial stripes.

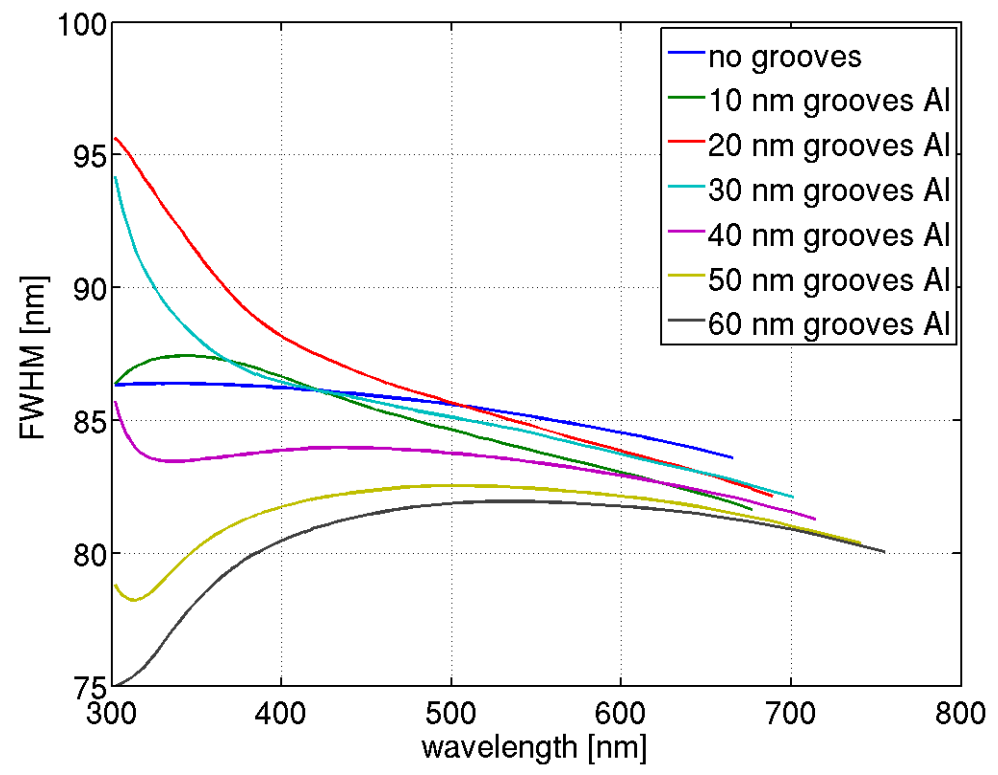

Figure 6. Full-width at half-maximum of the longitudinal field component $\mathrm{Hz}$ calculated $10 \mathrm{~nm}$ from the apex of a dielectric probe with silver stripes.

\section{CONCLUSIONS}

An advantage of the proposed magnetic field concentrator is that it may be employed as a scanning near-field probe with shear force control of the probe-sample distance. This distance of single tens of nanometers allows for illumination of individual elementary cells of an arrayed metamaterial. Linear dimensions of those cells should be slightly smaller than FWHM values $160 \mathrm{~nm}$ and $80 \mathrm{~nm}$ achievable for dielectric probes with radial metal stripes and dielectric probes with continuous metal coating and radial corrugations, respectively. Near-field illumination excites resonances in an elementary cell and $|\mathrm{E}| 2$ of scattered light is recorded with a square detector in the far-field.

\section{ACKNOWLEDGMENTS}

This work was sponsored by Polish grants from the National Science Centre \# 2011/01/M/St3/05734 and the National Centre for Research and Development \# N R15 0018 06/2009. The authors are partners in COST Action MP 0803. Simulations were performed at the Interdisciplinary Centre for Mathematical and Computational Modelling at the University of Warsaw under grant \#G33-7. 


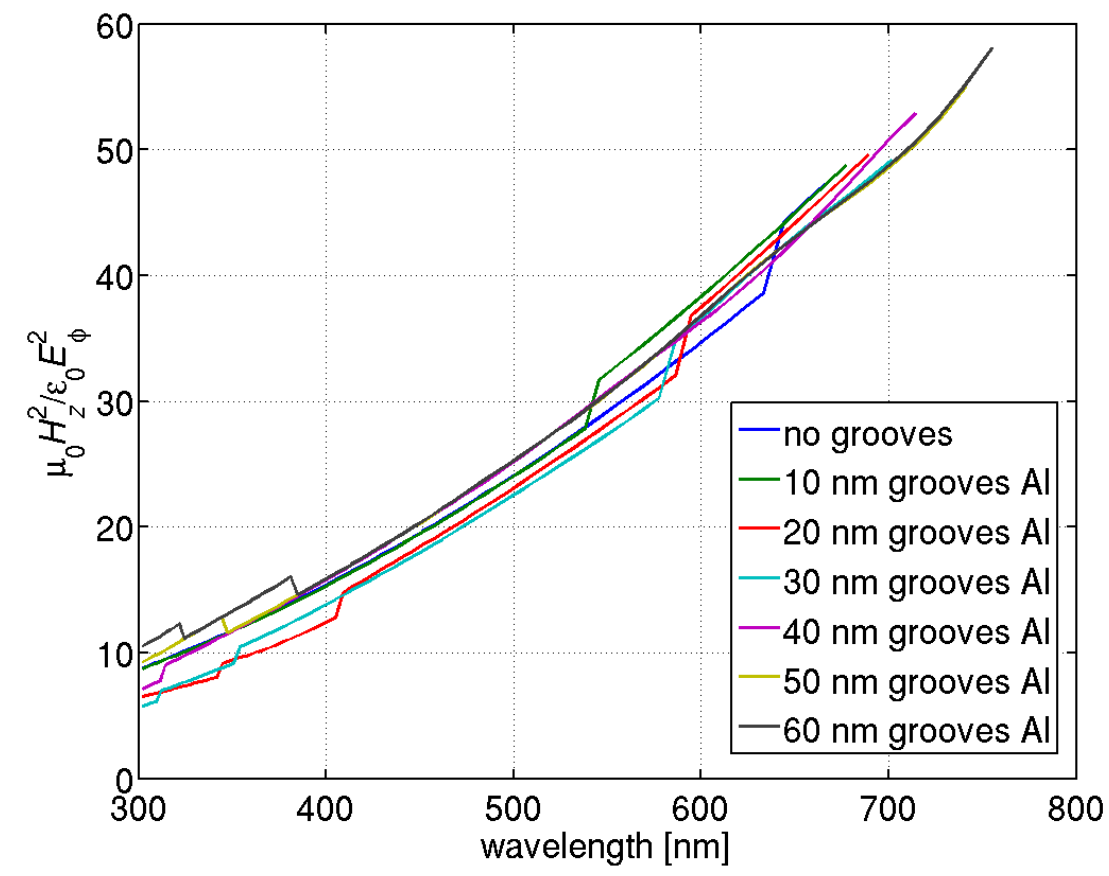

Figure 7 Ratio of magnetic energy density of $\mathrm{Hz}$ to electric energy density of $\mathrm{E} \varphi$ integrated over an area of diameter equal to the FWHM of $\mathrm{Hz}$ for dielectric probes with silver stripes.

\section{REFERENCES}

[1] J. Pendry, A. Holden, D. Robins, W. Stewart, "Magnetism from conductors and enhanced nonlinear phenomena," IEEE Trans. on Microwave Theory and Tech. 47, 2075-2084 (1999).

[2] Y.A. Urzhumov, G. Shvets, "Optical magnetism and negative refraction in plasmonic metamaterials," Solid State Commun. 146, 208-220 (2008).

[3] R. Merlin, "Metamaterials and the Landau-Lifshits permeability argument: large permittivity begets highfrequency magnetism," Proc. Natl. Acad. Sci. U.S.A. 106, 1693-1698 (2009).

[4] E. Devaux, A. Dereux, E. Bourillot, J.-C. Weeber, Y. Lacroute, and J.-P. Goudonnet, C. Girard, "Local detection of the optical magnetic field in the near zone of dielectric samples," Phys. Rev. B 62, 10504-10514 (2000).

[5] M. Burresi, D. van Oosten, T. Kampfrath, H. Schoenmaker, R. Heideman, A. Leinse, L., L. Kuipers, "Probing the magnetic field of light at optical frequencies," Science 326, 550-553 (2009).

[6] M. Burresi, T. Kampfrath, D. van Oosten, J. Prangsma, B. Song, S. Noda, S., L. Kuipers, "Magnetic light-matter interactions in a photonic crystal nanocavity," Phys. Rev. Lett. 105, 123901 (2010).

[7] S. Vignolini, F. Intonti, F. Riboli, L. Balet, L. Li, M. Francardi, A. Gerardino, A. Fiore, D. Wiersma, M. Gurioli, "Magnetic imaging in photonic crystal microcavities," Phys. Rev. Lett. 105, 123902 (2010).

[8] P. Banzer, U. Peschel, S. Quabis, G. Leuchs, "On the experimental investigation of the electric and magnetic response of a single nano-structure," Opt. Express 18, 10905-10923 (2010).

http://www.opticsinfobase.org/oe/abstract.cfm?URI $=0$ e-18-10-10905

[9] H.W. Kihm, S.M. Koo, Q.H. Kim, K. Bao, J.E. Kihm, W.S. Bak, S.H. Eah, C. Lienau, H. Kim, P. Nordlander, N.J. Halas, N.K. Park, and D.-S. Kim, "Bethe-hole polarization analyser for the magnetic vector of light," Nature Commun. 2, 1-6 (2011).

[10] T.J. Antosiewicz, P. Wróbel, T. Szoplik, "Magnetic field concentrator for probing optical magnetic metamaterials," Opt. Express 18, 25906-25911 (2010). http://www.opticsinfobase.org/oe/abstract.cfm?URI=oe-18-25-25906

[11] T. J. Antosiewicz, P. Wróbel, T. Szoplik, "Magnetic probe for material characterization at optical frequencies," Proc. SPIE 8070, 80700E-1 - 9 (2011). 
[12] J. S. Lee, S. Han, J. Shirdel, S. Koo, D. Sadiq, C. Lienau, N. Park, "Superfocusing of electric or magnetic fields using conical metal tips: effect of mode symmetry on the plasmon excitation method," Opt. Express 19, 1234212347 (2011). http://www.opticsinfobase.org/oe/abstract.cfm?URI=oe-19-13-12342

[13] P. Johnson, R. Christy, “Optical constants of the noble metals,” Phys. Rev. B 6, 4370-4379 (1972).

[14] M.A. Ordal, L.L. Long, R.J. Bell, R.R. Bell, R.W. Alexander, C.A. Ward, "Optical constants of themetals Al, Co, $\mathrm{Cu}$. Fe, Pb, Ni, Pd, Pt, Ag, Ti, and W in the infrared and far infrared," Appl. Opt. 22, 1099-1119 (1983). http://www.opticsinfobase.org/ao/abstract.cfm?URI=ao-22-7-1099

[15] P. Wróbel, T. Stefaniuk, T.J. Antosiewicz, A. Libura, G. Nowak, T. Wejrzanowski, R. Slesinski, K. Jedrzejewski, T. Szoplik, "Fabrication of corrugated probes for scanning near-field optical microscopy," Proc. SPIE 8070, 80700I-1-7 (2011).

[16] T. Antosiewicz and T. Szoplik, "Corrugated metal-coated tapered tip for scanning near-field optical microscope," Opt. Express 15, 10920-10928 (2007), http://www.opticsinfobase.org/oe/abstract.cfm?URI=oe-15-17-10920

[17] T.J. Antosiewicz, T. Szoplik, "Corrugated SNOM probe with enhanced energy throughput," Opto-Electron. Rev. $16,451-457$ (2008).

[18] T.J. Antosiewicz, P. Wróbel, T. Szoplik, "Performance of scanning near-field optical microscope probes with single groove and various metal coatings," Plasmonics 6, 11-18 (2011). 